\title{
ТРАНСДИАГНОСТИЧЕСКИЕ БИОМАРКЕРЫ В ОЦЕНКЕ СУИЦИДААЬНОЙ ПРЕДИСПОЗИЦИИ У НАРКОТИЧЕСКИХ АДДИКТОВ
}

\author{
С.В. Пронин, М.Г. Чухрова
}

ФГБОУ ВО «Новосибирский государственный педагогический университет», г. Новосибирск, Россия ФГБОУ ВО “Новосибирский государственный университет экономики и управления", г. Новосибирск

Контактная информация:

Пронин Сергей Владимирович - кандидат медицинских наук (SPIN-код: 7715-5812; ORCID iD: 0000-00022626-6021). Место работы и должность: доцент кафедры социальной психологии и виктимологии ФГБОУ ВО «Новосибирский государственный педагогический университет». Адрес: Россия, 630126, г. Новосибирск, ум. Вимюйская, 28; доцент кафедры психологии, педагогики и правоведения ФГБОУ ВО «Новосибирский государственный университет экономики и управления». Адрес: Россия, 630099, г. Новосибирск, ул. Каменская, 52/1. Телефон: (383) 244-00-95, эмектронный адрес: pronin53@gmail.com

Чухрова Марина Геннадьевна - доктор медицинских наук (SPIN-код: 4250-9594, AuthorID: 475871). Mесто работы и должность: профессор кафедры общей психологии и истории психологии ФГБОУ ВО “Новосибирский государственный педагогический университет". Адрес: Россия, 630126, г. Новосибирск, ул. Вилюйская, 28; профессор кафедры психологии, педагогики и правоведения ФГБОУ ВО “Новосибирский государственный университет экономики и управления". Адрес: Россия, 630099, г. Новосибирск, ул. Каменская, 52/1. Телефон: (383) 24400-95, эмектронный адрес: mba3@ngs.ru

Цель исследования: обоснование возможности неинвазивной приборной оценки суицидальной предиспозиции на основе показателей вариабельности сердечного ритма у лиц с наркотической зависимостью. Материал. В наркологическом стационаре был обследован 321 пациент с верифицированной по критериям ICD-X второй стадией зависимости (F11.xx2). Общее число пациентов, отметивших наркотические передозировки, составило 36,5\%. Разделение на группы контроль / основная проводилась с помощью опросника Бека. Первая (1) - контрольная группа - пациенты без проявлений аутоагрессивности (n=99 человек, в том числе 85 мужчин и 14 женщин, в возрасте 27,3 / 27,4 лет). Вторая (2) группа - сравнения - 222 пациента с аутоагрессивными проявлениями (мужчин - 148, женщин - 74; возраст: 27,3 / 25,8 лет). Общий балл на момент обращения по шкале Бека в первой группе - 10,5, во второй - 24,4 балла $(\mathrm{P}<0,01)$, что свидетельствует об умеренной депрессии среди пациентов группы 2. Сумма баллов по введённой нами субшкале «аутоагрессивность» в контрольной группе - 1,2; основной - 6,9 балла. Третья (3), референтная группа: здоровые лица (n=32) в возрасте $24,0 \pm 1,8$ лет. Результаты: сосудистый компонент адаптации преобладал в первой группе, где индекс LF: $42 \%$ (1) / 34\% (2) / 24\% (3) (P<0,05). Во второй группе доминировал нейроэндокринный компонент, где было такое распределение индекса VLF: 27\% / 55\% / 16\% (P<0,001). Высокочастотный - HF, (дыхательный) компонент был самым значительным в структуре ВСР среди группы здоровых лиц (гомеостатическое состояние): $31 \%$ / 11\% / $60 \%$ ( $<<0,001)$. Показатель общей мощности ВСР по индексу ТF у лиц с аутоагрессивностью превышал в 1,78 раза данный параметр пациентов контрольной группы и в 1,20 раза был выше по отношению к здоровым лицам. Диагностическая ценность спектральных характеристик BCP (VLF, LF, HF) в выявлении идеаторного компонента «суицидные мысли» составила $\mathrm{SE}=42 \%$; $\mathrm{SP}=100 \%$; преваленс $=75 \%$. Выводы. Трансдиагностический биомаркер - вариабельность сердечного ритма, его спектральные характеристики, могут быть ассоциированы с аутоагрессивной, суицидальной предиспозицией у лиц с аддиктивной патологией.

Ключевые слова: аутоагрессивность, суицидальная предиспозиция, наркотическая зависимость, алкоголизм, трансдиагностический биомаркер, гемодинамическая теория, вариабельность сердечного ритма, вегетативная нервная система

Объективная диагностика внутренней картины суицидального поведения нередко сложна $[1,2]$. Импульсивный суицидальный деликт может быть спровоцирован различными эндогенными, экзогенными и /или экологическими, факторами [3, 4, 5, 6]. Для наркологических аддиктов характерным является компонент суицидальной предиспозиции - внутренней готовности совершить суицид, который не проявляется в поведении, не вербализуется и, зачастую, не осознаётся ими [7]. Поэтому необходим поиск биомаркеров таких «внутренних» состояний, которые могут быть отнесены к триггерными механизмами потери контроля над своей жизнью, приводящего в итоге к саморазрушительному аутоагрессивному поведению, либо суициду. Ведущими в этой ситуации могут быть компоненты, связанные с дезадаптацией интеграции вегетативной и высшей нервной деятельности, дисбалансом систем 
внутреннего вознаграждения (ревард стимуляция), объясняющих изменённые состояния сознания и рисковое поведение в целом [8].

Известно, что сердечная деятельность находится под влиянием нейроэндокринной и вегетативной нервной системы, её симпатического и парасимпатического отделов. Дизадаптивность функционирования центральной нервной системы (ЦНС) опосредуется через вегетативную нервную систему (ВНС) и проявляется изменениями реактивности сердечно сосудистой системы, и это можно выявить при анализе вариабельности сердечного ритма (BCP). Деятельность ВНС, в частности, определяет индивидуальную способность к гибкому и адаптивному реагированию на внешние природные вызовы (магнитные бури, природные катаклизмы, социальные интеракции и т.п.) посредством модуляции симпатической / парасимпатической активности. Сохранение стабильности ВНС, как и её реактивность коррелируют со способностью регулировать негативные эмоции $[9,10]$, агрессивностью, тревожностью, гневливостью $[11,12]$, социальной адаптивностью [13], включая депрессивное [14, $15]$, биполярное расстройство $[16,17,18]$, пограничное расстройство личности $[19,20]$, генерализованое тревожное расстройство [21]. При этом ВСР подвержена влиянию различных неадаптивных психических состояний и последствий приёма алкоголя [22], а также реагирует на изменения уровня катехоламинов при быстрой опиоидной детоксикации [23], на лечение антипсихотиками [24]. Выявляется определённая взаимосвязь депрессивных и суицидальных идей и вариабельности сердечного ритма [25]. Также нами ранее была определена возможность прогнозирования психотических нарушений при состояниях отмены у лиц с различными формами «химической» зависимости на основе анализа некоторых параметров BCP [26].

Необходимо подчеркнуть, что возможность контролировать, осмысливать и интерпретировать данные регистрации ВСР зависят от технологии её записи, методики количественной оценки параметров сердечного ритма, знания ассоциированности основных нейрогуморальных механизмов, опосредующих изменения сердечного ритма с психологическими, физиологическими процессами в применении к конкретной поведенческой ситуации. В настоящее время предлагается считать ВСР трансдиагностическим тестом, и она рассматривает- ся как психофизиологический маркер общей психопатологии [27].

В совокупности, имеющиеся на сегодняшний день данные литературы [25, 27 и др.], позволяют говорить о влиянии активности ВНC на ВСР, где изменение её некоторых составляющих может быть ассоциировано с наличием суицидальных идей у пациентов.

Методология нашего исследования выстраивалась в рамках многопрофильной модели мониторинга наркомании, которая часто рассматривается как суицидальная предиспозиция [28]. Ранее нами [29] была установлена диагностическая важность гемодинамических показателей в оценке риска психотических нарушений, а также актуализации патологического влечения к психоактивным веществам (ПАВ).

Цель исследования: обоснование возможности неинвазивной приборной оценки суицидальной предиспозиции на основе показателей вариабельности сердечного ритма у лиц с наркотической зависимостью.

Материал и методы.

Дизайн исследования: открытое, ретроспективное, нерандомизированное. Критерии включения: 1) диагноз зависимости от ПАВ и состояние отмены, установленные в соответствии с критериями DSM-IV (1994), DSM-IVTR и ICD-X; 2) у пациентов на момент госпитализации по критериям ICD-X верифицирована вторая стадия зависимости (F11.xx2).

Было проведено комплексное психодиагностическое и инструментальное обследование 321 пациента с наркотической зависимостью с использованием шкалы депрессии Бека (Beck Depression Inventory).

К общим критериям исключения из исследования относились: 1) острые инфекционные заболевания; 2) наличие у пациентов эндогенных психических расстройств.

Пациенты разделены на две группы: первая (1), контрольная - лица без проявлений аутоагрессивности - состояла из 99 человек, в том числе 85 мужчин $(85,8 \%)$ и 14 женщин $(14,2 \%)$, в возрасте от 20 до 33 лет (средний у мужчин $-27,4 \pm 5,3$ у женщин $-25,6 \pm 4,6)$. Вторая (2) группа сравнения или основная, 222 пациента с аутоагрессивными проявлениями. Из них 148 мужчин (66,7\%), 74 женщины (33,3\%). Общий балл на момент обращения по шкале Бека составил в первой группе - 10,5 балла, второй $-24,4$ балла $(\mathrm{P}<0,01)$. 
Общая характеристика сравниваемых групп (n=321)

\begin{tabular}{|c|c|c|c|c|c|c|c|}
\hline \multicolumn{2}{|c|}{ Ковариация } & \multicolumn{3}{|c|}{$\begin{array}{c}\text { Группа (1) Контроль. } \\
\text { Без аутоагрессивности по } \\
\text { шкалам опросника Бека } \\
\end{array}$} & \multicolumn{3}{|c|}{$\begin{array}{c}\text { Группа (2) Основная. } \\
\text { Аутоагрессивность по шкалам } \\
\text { опросника Бека } \\
\end{array}$} \\
\hline Пол & Передозировки & $\mathrm{n}$ & $\begin{array}{l}\mathrm{M} \pm \mathrm{SD} \\
\text { лет }\end{array}$ & $\begin{array}{l}\text { \% внутри } \\
\text { групп }\end{array}$ & $\mathrm{n}$ & $\begin{array}{l}\mathrm{M} \pm \mathrm{SD} \\
\text { лет }\end{array}$ & $\begin{array}{l}\text { \% внутри } \\
\text { групп }\end{array}$ \\
\hline \multirow{3}{*}{ Мужчины } & да & 28 & $28,2 \pm 4,8$ & 32,9 & 52 & $27,9 \pm 5,6$ & 35,1 \\
\hline & нет & 57 & $26,9 \pm 5,5$ & 67,1 & 96 & $27,0 \pm 5,7$ & 64,9 \\
\hline & Всего & 85 & $27,4 \pm 5,3$ & 100,0 & 148 & $27,3 \pm 5,6$ & 100,0 \\
\hline \multirow{3}{*}{ Женщины } & да & 0 & - & 0 & 22 & $26,9 \pm 3,7$ & 29,7 \\
\hline & нет & 14 & $23,9 \pm 3,9$ & 100,0 & 52 & $23,0 \pm 3,4$ & 70,3 \\
\hline & Всего & 14 & $23,9 \pm 3,9$ & 100,0 & 74 & $24,2 \pm 3,9$ & 100,0 \\
\hline & Итого: & 99 & $25,6 \pm 4,6$ & & 222 & $25,8 \pm 4,8$ & \\
\hline \multicolumn{2}{|c|}{ Гепатит В и/или C } & & & 41,6 & & & 45,9 \\
\hline \multicolumn{2}{|c|}{ Отмечено наличие судимости } & 26 & $28,5 \pm 4,6^{* *}$ & 26,0 & 66 & $26,4 \pm 5,1$ & 29,6 \\
\hline \multicolumn{2}{|c|}{ Травма головы в анамнезе } & & & 38,8 & & & 37,8 \\
\hline \multicolumn{2}{|c|}{ Родители страдали алкоголизмом } & & & 23,9 & & & 19,6 \\
\hline \multicolumn{2}{|c|}{ Возраст начала приема наркотиков, лет } & & $20,8 \pm 5,3$ & & & $20,5 \pm 5,1$ & \\
\hline \multicolumn{2}{|c|}{$\begin{array}{l}\text { Общий балл депрессивных самооценок } \\
\text { по опроснику Бека (Д0) баллы }\end{array}$} & & $10,5 \pm 6,8$ & & & $24,4 \pm 8,0^{*}$ & \\
\hline \multicolumn{2}{|c|}{$\begin{array}{l}\text { Сумма баллов по шкалам Д+Ж+3+И } \\
\text { «аутоагрессивность» }\end{array}$} & & $1,2 \pm 0,1$ & & & $6,9 \pm 0,2$ & \\
\hline \multicolumn{2}{|c|}{$\begin{array}{l}\text { DSM-IV суммарный критерий зависимо- } \\
\text { сти (баллы) }\end{array}$} & & $5,8 \pm 1,2$ & & & $6,2 \pm 1,1$ & \\
\hline \multicolumn{2}{|c|}{ Средний балл толерантности к ПАВ } & & $14,5 \pm 12,3$ & & & $17,5 \pm 12,7 *$ & \\
\hline \multicolumn{2}{|c|}{ Индекс массы тела кг/м² } & & $23,3 \pm 3,5$ & & & $21,8 \pm 2,9$ & \\
\hline
\end{tabular}

Показатели центральной гемодинамики (ЦГД)

\begin{tabular}{l|c|c|c}
\hline АДС мм.рт.ст. & $119 \pm 13$ & $111 \pm 14$ & $0,036^{* *}$ \\
\hline АДД мм.рт.ст. & $80 \pm 10$ & $74 \pm 9$ & $0,015^{* *}$ \\
\hline Пульс уд. в мин. & $90 \pm 13$ & $88 \pm 9$ & 0,410 \\
\hline среднее АД мм.рт.ст. & $93 \pm 10$ & $87 \pm 10$ & $0,013^{* *}$ \\
\hline
\end{tabular}

Примечание: *P<0,0001 **P $<0,01$

Разделение на группы контроль / основная проводилась по введённой нами на основе опросника Бека интегральной шкале «аутоагрессивность». Суммировались баллы по следующим субшкалам: «Д» (чувство вины) + «Ж» (отвращение к самому себе) + «З» (идеи самообвинения) + «И» (суицидальные мысли). При сумме баллов $>4$, пациенты были отнесены к основной группе с «аутоагрессивностью». При меньшем количестве баллов, пациенты включались в группу «контроль». Средняя сумма баллов по шкале «аутоагрессивность» составила: контроль 1,2 балла / основная 6,9 балла.

Пациенты, у которых в анкетах не было отметок по этим шкалам, исключались из об- работки. Реперные точки «суицидные мысли «есть / нет» получены на основе соответствующей шкалы «И» опросника Бека.

Возраст начала приёма наркотиков в первой группе $20,8 \pm 5,3$, второй - 20,5 $\pm 5,1$ лет. Коморбидность - гепатит В и / или С в первой группе $-41,6 \%$, второй $-45,9 \%$. Травма головы в прошлом - 38,8 / 37,8\%. Наличие алкогольной зависимости у родителей - 23,9 / $19,6 \%$. Наличие судимости по статье 228 УК РФ: 29,0 / 29,6\%. Индекс массы тела - 23,3 / 21,8 кг/м² (табл. 1).

Была построена регрессионная модель связи вариабельности сердечного ритма её спектральных характеристик со всеми компонентами шкалы депрессии Бека. Уравнения были 
скорректированы по возрасту и полу. Окончательная модель получена с использованием пошаговой процедуры, поддерживающей переменные со значением ниже 0,10 .

Для исследования вегетативного баланса на основе ВСР применялся спектральный анализ сердечного ритма $[30,31]$. Исходные данные регистрировались с помощью программно - аппаратного комплекса «БОС - ПУЛЬС» (фотоплетизмографический датчик). Фоновая запись проводилась в условиях покоя в течение не менее 5 минут, которая осуществлялась в затемнённой комнате с периодом адаптации перед тестированием к окружающим условиям в течение 5-10 мин. Такая методика измерения демонстрирует высокую надёжность.

Записи ВСР выполнялись в промежутке с $9^{00}$ до $12^{00}$ часов дня, в комфортных условиях, при температуре воздуха в палате $20-22^{\circ} \mathrm{C}$. Перед этим исключался приём пищи. Качество записи проверялось с помощью коэффициента автокорреляции (ACF). По результатам данных ВСР оценивались такие спектральные индексы: VLF - медленные волны второго порядка, которые связаны с центральными эрготропными влияниями и отражают уровень психоэмоционального напряжения; LF - вазомоторные волны или волны 1-го порядка; HF - дыхательные волны; LF / HF - соотношение уровня центрального и автономного контура регуляции. Коэффициент вагосимпатического баланса, обычно повышен при активности симпатической нервной системы. Рассчитывался индекс централизации $\mathrm{IC}=(\mathrm{LF}+\mathrm{VLF}) / \mathrm{HF}-$ он показывает степень централизации управления ритмом сердца, то есть преобладание активности центрального контура регуляции над автономным.

Референтной (3) группой $(\mathrm{n}=32)$ для нормативного сравнения показателей ВСР послужили материалы исследования [32], проведённого нами среди здоровых лиц в возрасте $24,0 \pm 1,8$ лет (табл. 1).

Все пациенты в соответствии с принципами Хельсинской Декларации Всемирной Медицинской Ассоциации дали информированное письменное согласие на участие в обследовании. Материалы исследования были рассмотрены и получили одобрение на этической комиссии по месту работы авторов.

Методика статистического анализа.

В основе обработки данных - пакет SPSS

22. Применялся анализ методом ближайшего соседа - Nearest Neighbor Analysis. Проводилась оценка эффектов межгрупповых факторов - GLM Multivariate Analysis; факторный анализ; оценка площади под ROC кривыми; peгрессионный анализ (линейный и логистический); ANOVA дисперсионный анализ.

Результаты исследования.

Число лиц отметивших передозировки, которые связанны с внутривенным немедицинским приёмом наркотиков, составило $36,5 \%$. Заметна связь с возрастом пациентов $(\mathrm{F}=8,06$; $\mathrm{P}=0,005)$ и длительностью заболевания $(\mathrm{F}=10,14 ; \mathrm{P}=0,002)$. Возраст начала приёма наркотиков не имел такого влияния.

Анализ пригодности ступенчатых и дихотомных шкал (ответы «да / нет») опросника Бека проводился с помощью расчёта коэффициента альфы Кронбаха. Здесь не было выявлено отличий по информативности ответов. Уровень значимости всех шкал опросника Бека был практически одинаково высоким и составил 0,84 - для ступенчатых и 0,86 баллов в случае применения дихотомных оценок.

Отмечено, что использование характеристик спектрального анализа ВСР в разделении на группы контроль / основная (аутоагрессивность) обоснована, где пригодность шкал по альфа Кронбаха составила 0,80 / 0,78, и это является вполне приемлемым для использования данных показателей с целью выявления групп рискового поведения.

Определено, что наибольшее значение имеет такой индекс ВСР, как разница между «LF - HF» (значимость 0,75; макс. 1,0). Вероятность прогноза по этому индексу составляет 85\% для шкалы «суицидальная предиспозиция». При этом отмечена корреляция показателей центральной гемодинамики (ЦГД) и ВСР, например, отрицательная связь величины пульса со средним значением мощности ВСР.

Можно отметить при сравнении с нормативными показателями между группами (1), (2) и (3) такие градации индекса LF / HF = 1,37 / 2,81 / 0,39 $(\mathrm{P}=0,016)$. Похожие соотношения имел индекс централизации $\mathrm{IC}=2,24$ / 7,39 / 0,40 ( $\mathrm{P}=0,0001)$. Разница по мощности ритмов составила $\left(\mathrm{LF} \mathrm{Mc} \mathrm{m}^{2}-\mathrm{HF} \mathrm{Mc}^{2}\right)=270 \mathrm{Mc}^{2} / 901 \mathrm{Mc}^{2} /$ -1207 мс $^{2}(\mathrm{P}=0,002)$. Можно заключить, что в основной группе преобладал центральный контур регуляции над автономным, с высокой активностью симпатической нервной системы. Он превышал значения контрольной группы на $30 \%$ (табл. 2). 
Сравнение показателей спектрального анализа ВСР в основной, контрольной группе пациентов

Таблица 2 и среди здоровых лиц (n=353)

\begin{tabular}{|c|c|c|c|c|c|c|c|}
\hline \multirow{2}{*}{$\begin{array}{c}\text { Показатель } \\
\text { спектрального } \\
\text { анализа ВСР }\end{array}$} & \multicolumn{2}{|c|}{$\begin{array}{c}\text { Контрольная группа (1) } \\
\text { (n=99) }\end{array}$} & \multicolumn{2}{|c|}{$\begin{array}{c}\text { Основная группа (2) } \\
(\mathrm{n}=222)\end{array}$} & \multirow[t]{2}{*}{$P_{1-2}$} & \multicolumn{2}{|c|}{$\begin{array}{c}\text { Здоровые лица (3) } \\
\text { нормативные показатели } \\
(\mathrm{n}=32)[32]\end{array}$} \\
\hline & $\mathrm{M} \pm \mathrm{SD}$ & $\%$ & $\mathrm{M} \pm \mathrm{SD}$ & $\%$ & & $\mathrm{M} \pm \mathrm{SD}$ & $\%$ \\
\hline$\overline{\mathrm{VLF}, \mathrm{Mc}^{2}}$ & $630 \pm 149$ & 27 & $2270 \pm 1342$ & 55 & 0,010 & $525 \pm 131$ & 16 \\
\hline$\overline{L F} \mathrm{Mc}^{2}$ & $984 \pm 143$ & 42 & $1397 \pm 747$ & 34 & 0,482 & $773 \pm 153$ & 24 \\
\hline HF mc $^{2}$ & $718 \pm 143$ & 31 & $496 \pm 165$ & 11 & 0,693 & $1980 \pm 170$ & 60 \\
\hline TF $\mathrm{mc}^{2}$ & $2332 \pm 34$ & 100 & $4164 \pm 2255$ & 100 & 0,172 & $3277 \pm 561$ & 100 \\
\hline$\overline{\mathrm{LF} / \mathrm{HF}}$ & 1,37 & & 2,81 & & 0,016 & 0,39 & \\
\hline$\overline{\mathrm{IC}}$ & 2,24 & & 7,39 & & 0,0001 & 0,40 & \\
\hline $\mathrm{LFMc}^{2}-\mathrm{HFMc}^{2}$ & 270 & & 901 & & 0,002 & -1207 & \\
\hline
\end{tabular}

Выявлены статистически значимые отличия между первой и второй группами пациентов по такому показателю спектрального анализа ВCP, как VLF (нейрогенный компонент $\mathrm{P}=0,01$ ). Также пациенты первой группы имели следующие особенности: индекс TF был ниже в 1,78 раза по отношению к группе сравнения (2) и в 1,40 раза ниже по отношению к здоровым лицам (3).

Полученные индексы VLF, LF, HF использованы в построении модели для шкалы «И» по опроснику Бека (суицидальные мысли «есть нет»). Диагностическая значимость составила: чувствительность $\mathrm{SE}=42 \%$, специфичность $\mathrm{SP}=100 \%$, преваленс (распространённость в выборке) $=75 \%$. По шкале «Ж» (отвращение к самому себе) $\mathrm{SE}=67 \% ; \mathrm{SP}=0 \%$, преваленс $=94 \%$. Диагностические характеристики шкалы «3» (идея самообвинения) $\mathrm{SE}=100 \%, \mathrm{SP}=90 \%$, преваленс $=38 \%$.

Оказалось, что выбранные нами шкалы этого теста между собой значимо взаимосвязаны $(\mathrm{P}=0,0001)$. Вопрос о наличии у пациентов суицидных мыслей может ассоциироваться с чувством вины $\left(\mathrm{r}_{\mathrm{sp}}=0,160\right)$, идеями самообвинения $\left(\mathrm{r}_{\mathrm{sp}}=0,291\right)$ и отвращением к самому себе $\left(\mathrm{r}_{\mathrm{sp}}=0,383\right)$. Одновременно мы пытались уточнить, может ли структура потребления ПАВ влиять на приведенные выше самооценки наркотических аддиктов. Выяснилось, что проекция агрессивного реагирования «на себя» или на окружающих меняется в зависимости от того, сочетается ли употребление, например, героина и алкоголя. Факторная нагрузка составила для компонентов первой / второй модели $=0,072 / 0,757$ (максимальная оценка 1,0). Coчетанный приём ПАВ оказывал заметное влияние на формирование суицидальной предиспозиции.
Обсуждение результатов.

Для объяснения особенностей становления у лиц с аддиктивными состояниями суицидальной предиспозиции нами использована концепция аллостаза. Главными отличиями гомеостаза от аллостаза являются контрольные механизмы, которыми они оперируют. Гомеостаз работает по механизму обратной связи (feedback), а аллостаз - прямой (feedforward) [33]. Также рассматривалась теория ауто / аллопластического приспособления [34]. Исходя из этого, можно предположить, что при неадаптивном психическом состоянии - аутоагрессивности, вариабельность сердечного ритма и его мощность будет возрастать пропорционально силе стрессового воздействия. Это состояние аллостаза или аллопластичное приспособление. При адаптивной реакции на стресс, при сохраненной мощности вариабельности сердечного ритма, его изменчивость будет находиться в функциональном диапазоне - реакция гомеостаза. При выходе за нижний нормативный диапазон ВСР будет аутопластичный тип приспособления. Схема приспособительных реакций может выглядеть так: аутопластические $\leftrightarrow$ гоместатические $\leftrightarrow$ аллопластические состояния.

В первой группе - отмечались аутопластические процессы адаптации, вариабельность сердечного ритма была низкой. В этой группе преобладал сосудистый компонент адаптации (1) / (2) / (3) Lf = 42\% / 34\% / 24\% ( $<<0,05)$.

Во второй группе - пациентов с аутоагрессивностью - доминировали нейроэндокринные, центральные механизмы адаптации и вагальная регуляция была значительно снижена. И здесь было следующее распределение такого показателя ВCP, как VLF: 27\% / 55\% / 16\% $(\mathrm{P}<0,001)$. Можно предположить, что у лиц этой группы преобладали аллопластические 
процессы адаптации и наблюдалась значительная вариабельность сердечного ритма.

В третьей группе, здоровые лица, в структуре ВСР преобладал высокочастотный (дыхательный) компонент $\mathrm{HF}=31 \%$ / $11 \%$ / $60 \%$ $(\mathrm{P}<0,001)$ и это гомеостатический процесс саморегуляции (табл. 2).

Необходимо отметить, что при наличии идеаторного компонента «суицидальные мысли» отмечается снижение суммарного эффекта вегетативной регуляции у лиц с такими проявлениями. Наблюдается своеобразный дизрегуляторный энергодефицит, и становится объяснимым высокий процент аутоагрессивности среди наркотических аддиктов, выявленный нами ранее - 69,2\% [35]. При этом 35,2\% пациентов отмечали наркотические передозировки, что также можно считать скрытой аутоагрессивностью.

Диагностическая ценность спектральных характеристик ВСР в выявлении идеаторного компонента «суицидные мысли» составила $\mathrm{SE}=42 \%$; $\mathrm{SP}=100 \%$; преваленс $=75 \%$, что свидетельствует о надёжности предлагаемого метода.

Психометрическая оценка исходного состояния пациентов, на наш взгляд, должна быть направлена на выявление лиц с высоким риском суицидальной предиспозиции. Предлагаемая субшкала «аутоагрессивность» на основе опросника Бека, так же по нашему мнению, убедительна в своём применении. Об этом свидетельствуют высокие значения субшкалы по коэффициенту пригодности альфа Кронбаха $(0,80)$.

Полученные нами результаты исследования согласуются с данными S. Wilson [36] и D. Adolph [37] о том, что ряд индексов ВСР могут быть трансдиагностическими маркерами риска суицидального поведения. В работе Т. Forkmann [38] высказывается мнение, что снижение вагусной активности, преобладание её дезадаптивной лабильности является маркером когнитивной негибкости, заставляя пациентов смотреть на преодоление проблемных ситуаций только в «черных или белых» тонах, рассматривая самоубийство как единственный способ избежать невыносимой жизни.

Необходимо остановиться на факторах, которые могут влиять на характеристику полученных нами результатов. Во-первых, это гетерогенность выборки, где гендерное соотношение составило 6:1 мужчины / женщины, коморбидность «нет / да»-1,4:1. Эти различия нивелировались с помощью введения в статанализ переменных взвешивания по полу и т.п.
Во-вторых, так как влияние табакокурения полностью исключить было невозможно, оно было минимизировано у пациентов и контролировалось с помощью персонала.

В-третьих, существует проблема качества записи ВСР, где тестирование приходилось повторять в 10\% всех случаев из-за выявленных дефектов или невозможности провести исследование в определённый момент времени. При этом необходимо учитывать, что на результаты обследования в день поступления пациентов (Д 0 ) могут влиять токсические эффекты ПАВ, принятых ими перед госпитализацией. Поэтому делать записи ВСР нужно в первый - четвертый день лечения, но потом необходимо убирать влияние этого фактора на полученные результаты с помощью введения в статанализ переменной взвешивания.

В-четвертых, мы ограничились рассмотрением только спектральных характеристик ВСР, которые вполне приемлемы для коротких 5-10 минутных записей. Считается, что такие арифметические характеристики BCP как RMSSD, SDNN, CV\% уместнее рассматривать при суточных записях. Эта точка зрения на наш взгляд, требует углубленного изучения статистической значимости таких индексов при коротких записях ВСР.

Предлагаемый нами подход на основе гемодинамической теории к выявлению суицидальной предиспозиции у наркотических аддиктов перекликается с поливагальной теорией S. Porges [10]. Основная идея последней - ЦНС и ВНС реагируют на вызовы реального мира по принципу иерархии, а не по принципу баланса. Согласно этим обеим концепциям, записывая сигналы висцеромоторного блока (вариабельность сердечного ритма, частотные характеристики, показатели ЦГД) можно оценить состояние всех остальных систем человека, в том числе и прогнозировать психические и поведенческие составляющие.

Динамический контроль ВСР с учётом выявленных закономерностей позволяет выбирать соответствующие состоянию пациента коррекционные подходы и лечение. Оценка динамики состояний отмены и последующего абстинентного синдрома у лиц с «химической» зависимостью, построенная на ведущих клинических симптомах, состоянии центральной гемодинамики, принимая во внимание показатели ВСР, даёт возможность учитывать особенности проявления заболевания и адекватно выстраивать 
терапию с минимальным психическим и физическим дискомфортом для пациентов.

Использование важного положения гемодинамической теории о необходимости достижения гомеостатичного диапазона ЦГД и ВСР в процессе реабилитации «химических» аддиктов, по нашим наблюдениям, повышает её эффективность и позволяет предупреждать наркотические срывы. Также разработанные подходы, основанные на использовании биомаркера «вариабельность сердечного ритма», позволяют выявлять суицидальную предиспозицию на доклинических стадиях, прогнозировать её течение, и дают возможность организации динамического наблюдения и профилактики аллостатической дезадаптации, с возможностью ежедневного мониторинга психофизического состояния этих лиц.

Заключение. Необходимо подчеркнуть, что для «аддиктивных» личностей характерна

Литература:

1. Коцюбинский А.П., Гусева О.В., Бутома Б.Г. Суицидальное поведение психически больных: биопсихосоциальный подход. Социальная и клиническая психиатрия. 2018; 28 (2): 511.

2. Зотов П.Б., Уманский М.С., Юшкова О.В. Особенности диагностики суицидального поведения наркозависимых. Академический журнал Западной Сибири. 2016; 12 (6): 51-53.

3. Розанов В.А., Григорьев П.Е. Экологические факторы и суицидальное поведение человека. Суицидология. 2018; 9 (2): 30-49.

4. Уманский М.С. Мотивы и суицидальная динамика у больных алкоголизмом позднего возраста. Академический журнал $3 a-$ падной Сибири. 2013; 9 (4): 87-88.

5. Спадерова Н.Н., Хохлов М.С. Суицидальное и агрессивное поведение (обзор литературы). Девиантология. 2018; 2 (1): 36-47.

6. Harris E.C., Barraclough B., Suicide as an outcome for mental disorders. A meta-analysis. Br. J. Psychiatry. 1997; 170 (3): 205228.

7. Бисалиев Р.В., Вешнева С.А. Суицидальное поведение у аддиктов и их родственников. Астрахань: ФГБОУ ВПО АГТУ, 2013. $273 \mathrm{c.}$

8. Пронин С.В., Чухрова М.Г., Пронина Н.А. и авт. Биоинформационная оценка ревард системы в изучении интернет зависимости среди подростков. Доклад на 2-й Российско - китайской научно - практической конференции «Актуальные вопросы биопсихосоциальной реабилитации пациентов с аффективными расстройствами», 7 октября 2015 года, Томск. http: // present5.com/bioinformacionnye-aspekty-revard-sistemyv-izuchenii-internet-zavisimosti/

9. Appelhans B.M., Luecken L.J. Heart rate variability as an index of regulated emotional responding. Review of General Psychology. 2006; 10: 229-240. https:.doi.org/10.1037/1089-2680.10.3.229.

10. Porges S.W. The Polyvagal Perspective. Biol. Psychol. 2007; 74 (2): 116-143. doi: 10.1016/j.biopsycho.2006.06.009

11. Scott B.G., Weems C.F. Resting vagal tone and vagal response to stress: associations with anxiety, aggression, and perceived anxiety control among youths. Psychophysiology. 2014; 51 (8): 718-727.

12. Vogele C., Sorg S., Studtmann M., Weber H. Cardiac autonomic regulation and anger coping in adolescents . Biol. Psychol. 2010; 85 (3): 465-471.

13. Geisler F.C., Kubiak T., Siewert K., Weber H. Cardiac vagal tone is associated with social engagement and self-regulation. Biol. Psychol. 2013; 93 (2): 279-86. doi: 10.1016/j.biopsycho.2013.02.013 аутоагрессия. У больных наркоманией наблюдаются все виды агрессивного поведения, направленные как на внешний мир (гетеродеструкция), так и на собственную личность (аутодеструкция). Проведённое исследование обосновывает возможность объективной оценки суицидальной предиспозиции на основе показателей вариабельности сердечного ритма у лиц с наркотической зависимостью. Разработанный нами подход, наряду с высокой информативностью, является неинвазивной малозатратной методикой, которая доступна для её практической реализации при любом уровне медико - социальной помощи. Возможно применение данной системы диагностики аутоагрессивности и для выявления лиц со склонностью к рисковому аддиктивному поведению, например, в общеобразовательной школьной сети, высших учебных заведениях.

References:

1. Kocjubinskij A.P., Guseva O.V., Butoma B.G. Suicidal'noe povedenie psihicheski bol'nyh: biopsihosocial'nyj podhod [Suicidal behaviour of mental patients: a biopsychosocial approach]. Social'naja $i$ klinicheskaja psihiatrija [Social and clinical psychiatry]. 2018; 28 (2): 5-11. (In Russ)

2. Zotov P.B., Umansky M.S., Yushkova O.V. Diagnostic of suicidal behavior in drug addicts. Academic Journal of West Siberia. 2016; 12 (6): 51-53. (In Russ)

3. Rozanov V.A., Grigoriev P.E. Environmental factors and suicide behavior in human being. Suicidology. 2018; 9 (2): 30-49. (In Russ)

4. Umansky M.S. The motives and dynamics of suicide in patients with alcoholism later age. Academic Journal of West Siberia. 2013; 9 (4): 87-88. (In Russ)

5. Spaderova N.N., Khokhlov M.S. Suicidal and aggressive behavior (review). Deviant Behavior (Russia). 2018; 2 (1): 36-47. (In Russ)

6. Harris E.C., Barraclough B., Suicide as an outcome for mental disorders. A meta-analysis. Br. J. Psychiatry. 1997; 170 (3): 205228.

7. Bisaliev R.V., Veshneva S.A. Suicidal'noe povedenie u addiktov i ih rodstvennikov [Suicidal behavior in addicts and their relatives]. Astrahan': FGBOU VPO AGTU, 2013. 273 s. (In Russ)

8. Pronin S.V., Chuhrova M.G., Pronina N.A. i avt. Bioinformacionnaja ocenka revard sistemy v izuchenii internet zavisimosti sredi podrostkov [Bioinformatic evaluation of the revard system in the study of Internet addiction among adolescents]. Doklad na 2-j Rossijsko - kitajskoj nauchno - prakticheskoj konferencii «Aktual'nye voprosy biopsihosocial'noj reabilitacii pacientov s affektivnymi rasstrojstvami», 7 oktjabrja 2015 goda, Tomsk. http: // present5.com/bioinformacionnye-aspekty-revardsistemy-v-izuchenii-internet-zavisimosti/ (In Russ)

9. Appelhans B.M., Luecken L.J. Heart rate variability as an index of regulated emotional responding. Review of General Psychology. 2006; 10: 229-240. https:.doi.org/10.1037/1089-2680.10.3.229.

10. Porges S.W. The Polyvagal Perspective. Biol. Psychol. 2007; 74 (2): 116-143. doi: 10.1016/j.biopsycho.2006.06.009

11. Scott B.G., Weems C.F. Resting vagal tone and vagal response to stress: associations with anxiety, aggression, and perceived anxiety control among youths. Psychophysiology. 2014; 51 (8): 718-727.

12. Vogele C., Sorg S., Studtmann M., Weber H. Cardiac autonomic regulation and anger coping in adolescents . Biol. Psychol. 2010; 85 (3): 465-471.

13. Geisler F.C., Kubiak T., Siewert K., Weber H. Cardiac vagal tone is associated with social engagement and self-regulation. Biol. Psychol. 2013; 93 (2): 279-86. doi: 10.1016/j.biopsycho.2013.02.013 
14. Kemp A.H., Quintana D.S., Gray M.A., et al. Impact of depression and antidepressant treatment on heart rate variability: a review and meta-analysis. Biol. Psychiatry. 2010; 67 (11): 1067-1074.

15. Rottenberg J. Cardiac vagal control in depression: a critical analysis. Biol. Psychol. 2007; 74 (2): 200-211.

16. Chang H.A., Chang C.C., Tzeng N.S., et al. Heart rate variability in unmedicated patients with bipolar disorder in the manic phase. Psychiatry Clin. Neurosci. 2014; 68 (9): 674-682. doi: 10.1111/pcn. 12178

17. Henry B.L., Minassian A., Paulus M.P., et al. Heart rate variability in bipolar mania and schizophrenia. J. Psychiatr. Res. 2010; 44 (3): 168-176.

18. Moon E., Lee S.H., Kim D.H., Hwang B. Comparative study of heart rate variability in patients with schizophrenia, bipolar disorder, post-traumatic post-traumatic stress disorder, or major depressive disorder. Clin. Psychopharmacol. Neurosci. 2013; 11 (3): 137-143.

19. Austin M.A., Riniolo, T.C., Porges, S.W., Borderline personality disorder and emotion regulation: Insights from the Polyvagal Theory. Brain Cogn. 2007; 65 (1): 69-76.

20. Weinberg A., Klonsky E.D., Hajcak, G. Autonomic impairment in Borderline Personality Disorder: a laboratory investigation. Brain Cogn. 2009; 71 (3): 279-286.

21. Mankus A.M., Aldao A., Kerns C., et.al. Mindfulness and heart rate variability in individuals with high and low generalized anxiety symptoms. Behav. Res. Ther. 2013; 51 (7): 386-391.

22. Ingjaldsson J.T., Laberg J.C., Thayer J.F. Reduced heart rate variability in chronic alcohol abuse: relationship with negative mood, chronic thought suppression, and compulsive drinking. Biol. Psychiatry. 2003; 54 (12): 1427-1436. doi:10.1016/s00063223(02)01926-1

23. McDonald T., Hoffman W.E., Berkowitz R., et al. Heart rate variability and plasma catecholamines in patients during opioid detoxification. J Neurosurg. Anesthesiol. 1999; 11 (3): 195-199. doi:10.1097/00008506-199907000-00007

24. Khasawneh F.T., Shankar G.S. Adverse Effects of Atypical Antipsychotic Drugs in Patients with Schizophrenia. Cardiol. Res. Pract. 2014: 273060. doi: 10.1155/2014/273060.

25. Khandoker A.H., Luthra V., Abouallaban Y. et al. Predicting depressed patients with suicidal ideation from ECG recordings. Med. Biol. Eng. Comput. 2016; Aug 18. doi:10.1007/s11517-016-1557-y

26. Пронин С.В., Пронин В.С., Пронина Н.А. Анализ вариабельности сердечного ритма в прогнозе структуры потребления психоактивных веществ. Современные проблемы биологической психиатрии и наркологии. Вторая Всероссийская конференция с международным участием. Тезисы докладов (Томск, 4-6 марта 2008 г.) под научной ред. академика РАМН В.Я. Семке, проф. Т.П. Ветлугиной. Издательство «Иван Федоров». Томск, 2008. С. 190-192.

27. Beauchaine T.P., Thayer J.F. Heart rate variability as a transdiagnostic biomarker of psychopathology. Int. J. Psychophysiol. 2015; 98 (2 Pt 2): 338-350. doi: 10.1016/j.ijpsycho.2015.08.004

28. Бохан Н.А., Воеводин И.В., Мандель А.И. Научные основы первичной профилактики наркоманий. Актуальные вопросы пограничных и аффективных состояний. Томск-Барнаул, 1998. C. 33-35.

29. Пронина Н.А., Пронин С.В. Гемодинамические показатели в оценке эффективности лечения наркологических аддиктов. Реабилитация в наркологии: Материалы межрегиональной научно-практической конференции. Томск, 2002. С. 79-81.

30. Heart rate variability. Standards of measurement, physiological interpretation, and clinical use. Task Force of the European Society of Cardiology and the North American Society of Pacing and Electrophysiology. Europ Heart J. 1996; 17: 354-381.

31. Баевский Р.М., Иванов Г.Г., Чирейкин Л.В. и др. Анализ вариабельности сердечного ритма при использовании различных электрокардиографических систем: методические рекомендации. Вестник аритмологии. 2001; 24: 65-87.

32. Пронин С.В., Филиппова Ю.С., Чухрова М.Г. Новые возможности мониторинга состояния здоровья спортсменов, занимающихся в спортивных секциях, на основе анализа вариабельности сердечного ритма. VII Международный Научный Конгресс "Спорт, здоровье, человек" 27-29 октября 2015 г. г. Санкт-Петербург. Россия. Материалы Конгресса / Под ред. В.А. Таймазова. СПб; Изд-во «Олимп СПб». 2015. С. 219
14. Kemp A.H., Quintana D.S., Gray M.A., et al. Impact of depression and antidepressant treatment on heart rate variability: a review and meta-analysis. Biol. Psychiatry. 2010; 67 (11): 1067-1074.

15. Rottenberg J. Cardiac vagal control in depression: a critical analysis. Biol. Psychol. 2007; 74 (2): 200-211.

16. Chang H.A., Chang C.C., Tzeng N.S., et al. Heart rate variability in unmedicated patients with bipolar disorder in the manic phase. Psychiatry Clin. Neurosci. 2014; 68 (9): 674-682. doi: $10.1111 /$ pcn. 12178

17. Henry B.L., Minassian A., Paulus M.P., et al. Heart rate variability in bipolar mania and schizophrenia. J. Psychiatr. Res. 2010; 44 (3): 168-176.

18. Moon E., Lee S.H., Kim D.H., Hwang B. Comparative study of heart rate variability in patients with schizophrenia, bipolar disorder, post-traumatic post-traumatic stress disorder, or major depressive disorder. Clin. Psychopharmacol. Neurosci. 2013; 11 (3): 137-143.

19. Austin M.A., Riniolo, T.C., Porges, S.W., Borderline personality disorder and emotion regulation: Insights from the Polyvagal Theory. Brain Cogn. 2007; 65 (1): 69-76

20. Weinberg A., Klonsky E.D., Hajcak, G. Autonomic impairment in Borderline Personality Disorder: a laboratory investigation. Brain Cogn. 2009; 71 (3): 279-286.

21. Mankus A.M., Aldao A., Kerns C., et.al. Mindfulness and heart rate variability in individuals with high and low generalized anxiety symptoms. Behav. Res. Ther. 2013; 51 (7): 386-391.

22. Ingjaldsson J.T., Laberg J.C., Thayer J.F. Reduced heart rate variability in chronic alcohol abuse: relationship with negative mood, chronic thought suppression, and compulsive drinking. Biol. Psychiatry. 2003; 54 (12): 1427-1436. doi:10.1016/s00063223(02)01926-1

23. McDonald T., Hoffman W.E., Berkowitz R., et al. Heart rate variability and plasma catecholamines in patients during opioid detoxification. J Neurosurg. Anesthesiol. 1999; 11 (3): 195-199. doi:10.1097/00008506-199907000-00007

24. Khasawneh F.T., Shankar G.S. Adverse Effects of Atypical Antipsychotic Drugs in Patients with Schizophrenia. Cardiol. Res. Pract. 2014: 273060. doi: 10.1155/2014/273060.

25. Khandoker A.H., Luthra V., Abouallaban Y., et al. Predicting depressed patients with suicidal ideation from ECG recordings. Med. Biol. Eng. Comput. 2016; Aug 18. doi:10.1007/s11517-016-1557-y

26. Pronin S.V., Pronin V.S., Pronina N.A. Analiz variabel'nost serdechnogo ritma $\mathrm{v}$ prognoze struktury potreblenija psihoaktivnyh veshhestv [Analysis of heart rate variability in the prognosis of the structure of psychoactive substances consumption]. Sovremennye problemy biologicheskoj psihiatrii i narkologii. Vtoraja Vserocsijskaja konferencija s mezhdunarodnym uchastiem. Tezisy dokladov (Tomsk, 4-6 marta 2008 g.) pod nauchnoj red. akademika RAMN V.Ja. Semke, prof. T.P. Vetluginoj. Izdatel'stvo «Ivan Fedorov». Tomsk, 2008. S. 190-192. (In Russ)

27. Beauchaine T.P., Thayer J.F. Heart rate variability as a transdiagnostic biomarker of psychopathology. Int. J. Psychophysiol. 2015; 98 (2 Pt 2): 338-350. doi: 10.1016/j.ijpsycho.2015.08.004

28. Bohan N.A., Voevodin I.V., Mandel' A.I. Nauchnye osnovy pervichnoj profilaktiki narkomanij [Scientific basis of primary drug abuse prevention]. Aktual'nye voprosy pogranichnyh i affektivnyh sostojanij. Tomsk-Barnaul, 1998. S. 33-35. (In Russ)

29. Pronina N.A., Pronin S.V. Gemodinamicheskie pokazateli v ocenke jeffektivnosti lechenija narkologicheskih addiktov [Hemodynamic parameters in assessing the effectiveness of treatment of drug addicts]. Reabilitacija v narkologii: Mat. mezhregional'noj nauchnoprakticheskoj konferencii. Tomsk, 2002. S. 79-81. (In Russ)

30. Heart rate variability. Standards of measurement, physiological interpretation, and clinical use. Task Force of the European Society of Cardiology and the North American Society of Pacing and Electrophysiology. Europ Heart J. 1996; 17: 354-381.

31. Baevskij R.M., Ivanov G.G., Chirejkin L.V. i dr. Analiz variabel'nosti serdechnogo ritma pri ispol'zovanii razlichnyh jelektrokardiograficheskih sistem: metodicheskie rekomendacii [Analysis of heart rhythm variability when using various electrocardiographics systems: guidelines]. Vestnik aritmologii [Bulletin of Arrhythmology]. 2001; 24: 65-87. (In Russ)

32. Pronin S.V., Filippova Ju.S., Chuhrova M.G. Novye vozmozhnosti monitoringa sostojanija zdorov'ja sportsmenov, zanimajushhihsja v sportivnyh sekcijah, na osnove analiza variabel'nosti serdechnogo ritma [New opportunities for monitoring the health of athletes involved in sports sections, based on the analysis of heart rate variability]. VII Mezhdunarodnyj Nauchnyj Kongress "Sport, zdorov'e, chelovek" 27-29 oktjabrja 2015 g. g. Sankt-Peterburg. Rossija. Materialy Kongressa / Pod red. V.A. Tajmazova. SPb; Izd-vo «Olimp 
220

33. http://en.wikipedia.org/wiki/Allostasis, http://sundorog. livejournal. com/47141.html).

34. Аллопластическая и аутопластическая адаптация. Национальная психологическая энциклопедия. http://vocabulary.ru/termin/alloplasticheskaja-iautoplasticheskaja-adaptacija.html

35. Пронин С.В., Баданова Т.С., Карпачев Д.А., Пронин В.С Предикторы аутоагрессивного поведения у лиц с наркотической зависимостью. Суицидология. 2011; 2 (1): 42-46.

36. Wilson S.T., Chesin M., Fertuck E., Keilp J., et al. Heart rate variability and suicidal behavior. Psychiatry Res. 2016; 30 (240): 241-247. doi: 10.1016/j.psychres.2016.04.033.

37. Adolph D., Teismann T., Forkmann T., et al. High frequency heart rate variability: Evidence for a transdiagnostic association with suicide ideation. Biol. Psychol. 2018; 22 (138): 165-171. doi: 10.1016/j.biopsycho.2018.09.006.

38. Forkmann T., Meessen J., Teismann, T., et al. Resting vagal tone is negatively associated with suicide ideation. Journal of Affective Disorders. 2016; 194: 30-32.
SPb». 2015. S. 219-220. (In Russ)

33. http://en.wikipedia.org/wiki/Allostasis, http://sundorog. livejournal. com/47141.html).

34. Alloplasticheskaja i autoplasticheskaja adaptacija. Nacional'naja psihologicheskaja jenciklopedija [Alloplastic and autoplastic adaptation. National psychological encyclopedia] http://vocabulary.ru/termin/alloplasticheskaja-i-autoplasticheskajaadaptacija.html (In Russ)

35. Pronin S.V., Badanova T.S., Karpachev D.A., Pronin V.S. Predictors of self-injurious behavior in drug abusers. Suicidology. 2011; 2 (1): 42-46. (In Russ)

36. Wilson S.T., Chesin M., Fertuck E., Keilp J., et al. Heart rate variability and suicidal behavior. Psychiatry Res. 2016; 30 (240): 241-247. doi: 10.1016/j.psychres.2016.04.033.

37. Adolph D., Teismann T., Forkmann T., et al. High frequency heart rate variability: Evidence for a transdiagnostic association with suicide ideation. Biol. Psychol. 2018; 22 (138): 165-171. doi: 10.1016/j.biopsycho.2018.09.006.

38. Forkmann T., Meessen J., Teismann, T., et al. Resting vagal tone is negatively associated with suicide ideation. Journal of Affec tive Disorders. 2016; 194: 30-32.

\title{
TRANSDIAGNOSTIC BIOMARKERS IN THE EVALUATION OF SUICIDAL PREDISPOSITION IN DRUG ADDICTS
}

\author{
S.V. Pronin 1,2 , M.G. Chuhrova ${ }^{1,2}$ \\ ${ }^{1}$ Novosibirsk State Pedagogical University, Novosibirsk, Russia; pronin53@gmail.com \\ ${ }^{2}$ Novosibirsk State University of Economics and Management, Novosibirsk, Russia; mba3@ngs.ru
}

\begin{abstract}
:
Objective: to substantiate the possibility of non-invasive instrument assessment of suicidal pre-disposition based on heart rate variability in people with drug addiction. Materials and methods. In the narcological hospital, 321 patients were examined with ICD-X-verified second stage of addiction (F11.xx2). The total number of patients who noted narcotic overdose was $36.5 \%$. The division into control / main groups was carried out using the Beck questionnaire. The first (1) - control group consisted of patients without manifestations of auto-aggressiveness $(n=99$ people, including 85 men and 14 women), at the age of 27.3 / 27.4. The second (2) group - comparisons - included 222 patients with auto-aggressive manifestations (men - 148, women - 74. Age: 27.3 / 25.8). The total score at the time of treatment on the Beck scale in the first group was 10.5 , in the second it was 24.4 points $(\mathrm{P}<0.01)$, which indicates moderate depression among the patients of group 2. The total points for the autoaggression subscript we entered in the control group was 1.2 and in the main it was 6.9 points. The third (3) reference group was made up of healthy individuals $(n=32)$ at the age of $24.0 \pm 1.8$. Results: the vascular component of adaptation prevailed in the first group, with the LF index of $42 \%$ (1) / 34\% (2) / 24\% (3) $(\mathrm{P}<0.05)$. The second group was dominated by the neuro-endogenous component, where there was such a distribution of the VLF index: 27\% / 55\% / 16\% (P <0.001). The high-frequency $\mathrm{HF}$, (respiratory) component was the most significant in the structure of HRV among the group of healthy individuals (homeostatic state): $31 \%$ / 11\% / 60\% ( $\mathrm{P}<0.001)$. The index of total HRV power on the TF index in individuals with auto-aggressiveness exceeded this parameter in control patients 1.78 times and was 1.20 times higher than in healthy individuals. The diagnostic value of the spectral characteristics of HRV (VLF, LF, HF) in identifying the ideological component "suicidal thoughts" was $\mathrm{SE}=42 \%$; $\mathrm{SP}=100 \%$; prevalence $=75 \%$. Conclusion. Trans diagnostic biomarker is heart rate variability, its spectral characteristics may be associated with auto-aggressive, suicidal predisposition in patients with addictive pathology.
\end{abstract}

Keywords: autoaggression, suicidal predisposition, drug addiction, alcoholism, transdiagnostic biomarker, hemodynamic theory, heart rate variability, autonomic nervous system

Финансирование: Исследование не имело финансовой поддержки.

Конфмикт интересов: Авторы заявляют об отсутствии конфмикта интересов.

Для цитирования: Пронин С.В., Чухрова М.Г. Трансдиагностические биомаркеры в оценке суицидальной предиспозиции у наркотических аддиктов. Суицидология. 2018; 9 (4): 109-117. doi.org/10.32878/suiciderus.18-09-04(33)-109-117

For citation: $\quad$ Pronin S.V., Chuhrova M.G. Transdiagnostic biomarkers in the evaluation of suicidal predisposition in drug addicts. Suicidology. 2018; 9 (4): 109-117. (In Russ) doi.org/10.32878/suiciderus.18-09-04(33)-109-117 